\title{
Summary
}

It would be very advantageous that the useful microorganismes, which are destroyed by antibiotics, should be provided to the human body by food. Cheeses might be this food, because their microbial flora is important and various.

\section{AURONS-NOUS DU BIO-FROMAGE? (1)}

\author{
par \\ ANDRÉ MOLDAVAN
}

Lors du récent congrès, à Montréal, de l'Association des Médecins de langue française de l'Amérique, le $D^{r}$ René Dubos, de l'Institut Rockefeller, dans sa conférence sur les antibiotiques faisait trois remarques d'un intérêt spécial pour l'industrie laitière.

1. - Certains germes pathogènes, en présence d'antibiotiques, se transforment en souches nouvelles résistantes à ces antibiotiques. Ces persisteurs perdent temporairement leur virulence mais restent une source possible d'infections ultérieures.

2. - Les antibiotiques détruisent spécifiquement certains germes pathogènes dans le corps mais en même temps, attaquent un grand nombre de microbes utiles à la santé, particulièrement ceux des intestins. Il en résulte une diminution de la résistance naturelle du corps suivie parfois d'infections gastro-intestinales secondaires.

3. - Le $\mathrm{D}^{\mathrm{r}}$ Dubos a exprimé l'espoir que la microbiologie s'efforcera d'identifier et de cultiver ces microbes utiles et bienfaisants afin de pouvoir les ensemencer à nouveau dans les intestins du patient après le traitement antibiotique.

\section{Microbiologie intestinale}

Nos connaissances de la microbiologie pathologique des intestins sont beaucoup plus vastes que celles de la microbiologie naturelle des intestins en bonne santé.

L'estomac et le duodénum de l'intestin grêle ont une population microbienne relativement faible, mais celle-ci augmente dans le jéjunum et dans l'iléum. Le colon ou gros intestin contient une

(1) Le Québec laitier, janvier 1963. 
flore et une faune microbienne extrêmement variées et luxuriantes. Ces microbes se développent sur une masse molle constituée par du mucus, des cellules mortes de la paroi intestinale et des aliments non digérés.

D'après RETTGER, la multiplication microbienne dans le colon normal peut atteindre cent mille milliards de cellules par jour, d'un poids total de 8 à 10 grammes.

Les actions et réactions biochimiques de ces microbes sont excessivement complexes. En plus des phénomènes de la reproduction cellulaire, de la symbiose, de l'antibiose et de la bactériophagie, il y a le jeu extraordinaire et surimposé de milliers d'enzymes, de substances organiques, de sécrétions glandulaires, de sucs digestifs et de sels minéraux, le tout formant un mélange inextricable soumis aux remous et au malaxage des mouvements péristaltiques et constrictoires et sujet à l'absorption des liquides par les parois intestinales.

A cela il faut ajouter les facteurs psychomatiques de l'hôte et leurs effets sur le système nerveux, musculaire et glandulaire et indirectement sur la digestion.

La classification des microbes intestinaux dépasse le cadre de cet article. D'une manière générale le groupe Escherichia coli prédomine. Les intestins du bébé nourri au lait maternel contiennent principalement du Lactobacillus bifidus qui est remplacé en partie par du Lactobacillus acidophilus à mesure que l'enfant reçoit du lait de vache.

\section{Interprétation de Brochu, Riel et Vézina}

La meilleure façon de résumer nos connaissances sur l'action des bactéries lactiques dans le système digestif est de citer textuellement les observations récentes de E. Brocho, R. RIEL et C. VÉzIna.

“De toutes les explications fournies depuis МЕтснкпкоFF (Institut Pasteur, 1904), sur les bienfaits des laits fermentés, très peu ont survécu à la rigueur de l'analyse scientifique et la plupart sont tombées faute de preuves expérimentales. L'hypothèse de l'hygiène intestinale, assurée par l'ingestion massive de laits fermentés a le mieux résisté. La synthèse de vitamines et la sécrétion d'antibiotiques par les bactéries lactiques ne semblent pas jouer un très grand rôle. La digestibilité accrue des protéines des laits fermentés, offre, cependant, une explication. Le lait contient des quantités bien équilibrées de tous les acides aminés : dans l'organisme normal, ces acides aminés sont libérés et absorbés par l'organisme. La fermentation par les bactéries lactiques produit un caillé 
plus fin et plus rapidement attaquable par les enzymes digestives, ce qui facilite la digestion et l'absorption des protéines du lait sous forme d'acides aminés. ”

\section{Sélection des microbes intestinaux bienfaisants}

Le sondage des intestins et la culture des spécimens au laboratoire permet d'identifier un très grand nombre d'espèces microbiennes et même de déterminer avec précision leur souche ou leur sérotype spécifique parmi les centaines ou milliers de variétés d'une seule espèce.

Mais l'étude la plus systématique au laboratoire n'est jamais un duplicata exact des conditions naturelles des intestins. De plus, il n'y a pas deux individus sur la terre qui réagissent physiologiquement de manière identique et absolument comparable.

Les travaux de BRochu, RIEL et V́́zina ont établi qu'il n'est pas suffisant d'ensemencer les intestins avec une microflore spécifique et favorable mais qu'il faut fournir en même temps la nourriture la mieux adaptée à cette multiplication microbienne.

La tâche scientifique envisagée par R. DuBos, celle d'une sélection de tous les microbes bienfaisants au corps humain est vraiment herculéenne mais concevable.

\section{Y a-t-il une solution naturelle?}

Il ne faut pas oublier que les vaccins, sérums, antibiotiques, enzymes et cryptogènes utilisés en microbiologie sont des substances naturelles que le savant finit par découvrir après de longues études. Plusieurs des antibiotiques les plus puissants doivent leur origine à des microbes du fumier et des eaux d'égouts.

Les hommes de science lorsqu'ils confrontent un problème de haute magnitude se demandent s'il n'y a pas moyen de contourner les difficultés scientifiques et de résoudre ce problème, temporairement, par des méthodes empiriques, plus élémentaires peut-être mais plus immédiates.

A ce propos, il est bon de se souvenir qu'un nutritionniste célèbre a résolu un problème très compliqué avec une solution aussi simple que limpide. Lord (Sir John) Boyd-ORR, premier président de l'organisation mondiale des aliments et de l'agriculture (F.A.O.) à qui on demandait de formuler un mélange de toutes les vitamines nécessaires à l'être humain : "Eat a little of everything and let the vitamins take care of themselves $n$.

Existe-t-il une substance naturelle qui contient déjà un grand nombre d'espèces microbiennes bienfaisantes, à leur optima de 
viabilité et d'action? Pourrait-on offrir au système digestif du patient un grand choix de microbes et se fier à la sélection naturelle pour l'ensemencement microbien des intestins "sanitisés " par les antibiotiques.

\section{Microbes des fromages}

De tous les aliments consommés par l'homme, ce sont les fromages à ensemencement naturel qui possèdent la flore microbienne la plus riche et la plus variée. Les éléments nutritifs des fromages aux stades successifs de la préparation et de la maturation, constituent un substratum particulièrement bien adapté à la croissance des microbes intestinaux.

Il faut évidemment, différencier entre les fromages de fabrique à cultures pures et les fromages de ferme, exposés à l'ensemencement microbien " naturel » des lieux.

L'histoire du Camembert est un bon exemple à rappeler. Marie Fontaine-Harel (1791) fut la première à fabriquer du Camembert et fut tôt imitée par les fermières voisines. Il y a un demi-siècle encore, on pouvait, sur les marchés de Normandie, se procurer côte à côte, des douzaines de variétés de Camembert, dont la différence en saveur, nul doute, provenait de souches microbiennes variant d'une ferme à l'autre.

Le livre Cheese Varieties (U.S. Dept of Agric.) décrit plus de 400 fromages types d'Europe et d'Amérique, qui diffèrent non seulement par leur origine et méthodes de fabrication, mais aussi par la nature des microbes qui s'y développent aux stades successifs de la maturation.

DuClaux, élève de Pasteur, fut le premier à étudier les microbes des fromages. Malheureusement, ses successeurs se sont surtout appliqués à sélectionner des cultures pures plutôt qu'à étudier les espèces microbiennes naturelles des fromages et leur rôle dans les intestins.

Les genres et espèces microbiens et leurs nombreuses souches trouvées dans le lait et les fromages appartiennent aux groupes suivants :

Bactéries : Pseudomonas, Micrococcus, Sarcina, Streptococcus, Leuconostoc, Lactobacillus, Microbacterium, Propionibacterium, Butyribacterium, Alcaligenes, Achromobacter, Flavobacterium, Escherichia, Aerobacter, Serratia, Bacterium, Brevibacterium, Bacillus.

Moisissures : Penicilium, Aspergillus, Geotrichum.

Levures : Saccharomyces, Candida, Torulopsis. 


\title{
Bio-Fromage
}

Dans la recherche antibiotique, le plus grand nombre possible de substances et de microbes sont examinés, sélectionnés, analysés et comparés. Les essais cliniques favorables ou défavorables sont le critère final de la valeur préventive ou curative de l'antibiotique.

La sélection d'un fromage ou d'une combinaison de fromages ayant les qualités désirables à l'ensemencement microbien des intestins "sanitisés" par les antibiotiques, serait plus empirique puisque l'effet en vue est constructif plutôt que destructif.

L'étude comparée des microbes intestinaux elt nettement bienfaisants, et des microbes obtenus du plus grand nombre possible de fromages "naturels ", à leur optima d'activité microbienne, permettrait une sélection initiale.

Les essais chimiques, avenant des résultats positifs et favorables, mesurés par l'absence d'infections gastro-intestinales secondaires, serviraient à une sélection progressive des genres de bio-fromages de réfection les plus effectifs.

Ce serait alors la tâche des experts fromagers de créer un biofromage spécifique en partant des cultures microbiennes sélectionnées par les microbiologistes.

\section{Summary}

Drinkable milk presents a great interest for Hygiene and Economy. It must taste nice and be sold under low temperature, it can be drunk easily at very moment during the day. One must be accustomed early to drink milk.

\section{SUPPLEMENT TECHNIQUE}

\section{L'ÉLECTRODIALYSE ET SES APPLICATIONS DANS L'INDUSTRIE LAITIÈRE}

\author{
par \\ G. GÉNIN \\ Ingénieur E.P.C.I.
}

On sait que l'électrodialyse est un procédé dans lequel il y a transfert d'électrolytes au travers d'une membrane, sous l'action d'un courant électrique. 